\title{
When Does Spatial Diversification Usefully Maximize the Durability of Crop Disease Resistance?
}

\author{
Benjamin Watkinson-Powell, Christopher A. Gilligan, and Nik J. Cunniffe ${ }^{\dagger}$ \\ Department of Plant Sciences, University of Cambridge, Downing St., Cambridge, CB2 3EA, United Kingdom \\ Accepted for publication 3 June 2020.
}

\begin{abstract}
Maximizing the durability of crop disease resistance genes in the face of pathogen evolution is a major challenge in modern agricultural epidemiology. Spatial diversification in the deployment of resistance genes, where susceptible and resistant fields are more closely intermixed, is predicted to drive lower epidemic intensities over evolutionary timescales. This is due to an increase in the strength of dilution effects, caused by pathogen inoculum challenging host tissue to which it is not well-specialized. The factors that interact with and determine the magnitude of this spatial suppressive effect are not currently well understood, however, leading to uncertainty over the pathosystems where such a strategy is most likely to be cost-effective. We model the effect on landscape scale disease dynamics of spatial heterogeneity in the arrangement of fields planted with either susceptible or resistant cultivars, and the way in which this effect depends on the parameters governing the pathosystem of interest. Our multiseason semidiscrete epidemiological model tracks spatial spread of wild-type and resistance-breaking pathogen strains, and incorporates a localized reservoir of inoculum, as well as the effects of within and between field transmission. The pathogen dispersal
\end{abstract}

ABSTRAC characteristics, any fitness cost(s) of the resistance-breaking trait, the efficacy of host resistance, and the length of the timeframe of interest all influence the strength of the spatial diversification effect. A key result is that spatial diversification has the strongest beneficial effect at intermediate fitness costs of the resistance-breaking trait, an effect driven by a complex set of nonlinear interactions. On the other hand, however, if the resistance-breaking strain is not fit enough to invade the landscape, then a partially effective resistance gene can result in spatial diversification actually worsening the epidemic. These results allow us to make general predictions of the types of system for which spatial diversification is most likely to be cost-effective, paving the way for potential economic modeling and pathosystem specific evaluation. These results highlight the importance of studying the effect of genetics on landscape scale spatial dynamics within host-pathogen disease systems.

Keywords: crop disease, durable resistance, evolutionary epidemiology, gene-for-gene system, mathematical modeling, optimal deployment, spatial dynamics, spatial heterogeneity
The evolution of pathogen populations in response to the use of resistant crop cultivars is a major area of concern for global food production. There is also environmental concern, as a lower efficacy of resistant crops generally leads to a greater reliance on chemical pesticides, with potential negative impacts on nonagricultural ecosystems. Increasing the durability of crop disease resistance, defined as the period of time that resistance remains effective with its widespread cultivation, is therefore an important focus for plant breeders and epidemiologists (Johnson 1979). Resistances can break down in a matter of years or even months, with resistances to fungal and bacterial diseases generally breaking down faster than those for viruses, in part due to the often lower mutational fitness costs in cellular pathogens (Garcia-Arenal and McDonald 2003). The resultant effect of this breakdown can be a significant reduction in crop yields, underlining the need to understand how host resistances can be effectively deployed.

The interaction between plant disease resistance and corresponding pathogen virulence, defined here as the qualitative ability of a pathogen to infect a host, often takes the form of a gene-for-gene

${ }^{\dagger}$ Corresponding author: N. J. Cunniffe; njc1001@cam.ac.uk

Funding: B. Watkinson-Powell acknowledges the Biotechnology and Biological Sciences Research Council of the United Kingdom (BBSRC) for support, via a University of Cambridge DTP Ph.D. studentship.

*The $e$-Xtra logo stands for "electronic extra" and indicates there are supplementary materials published online.

The author(s) declare no conflict of interest.

Copyright (C) 2020 The Author(s). This is an open access article distributed under the CC BY 4.0 International license. relationship (Flor 1971). This genetic system is defined by a single major $R$ gene in the host that recognizes, and provides qualitative resistance to, a single corresponding avirulence gene in the pathogen. Often this avirulence gene has an important role as an effector in the mechanism of infection (Cui et al. 2015). Mutations in the avirulence gene can cause it to evade the corresponding $R$ gene and become resistance-breaking or virulent (Flor 1971).

Major gene qualitative resistance in some crops has historically broken down relatively rapidly, and has been described as exhibiting boom-and-bust dynamics as new cultivars need to be repeatedly developed (McDonald and Linde 2002). The Mla13 resistance gene to powdery mildew on barley was overcome within 6 to 7 years after its introduction in the early 1980s (Wolfe and McDermott 1994), while the LrI1 and Lr24 winter wheat leaf rust resistance genes were overcome within 1 to 2 years (Kolmer 1996). In contrast, other major resistance genes have remained durable over very long periods of time. One notable example is the $\mathrm{Sr} 26$ resistance gene for wheat stem rust which has maintained its effectiveness ever since its development in 1961 (Knott 1961; Qureshi et al.2018). This is one of the few genes that resists the stem rust pathogen race TTKSK (Ug99) along with all other races of this pathogen (Qureshi et al. 2018).

There is substantial evidence that resistance-breaking traits in plant pathogens can have high reproductive fitness costs, with examples in plant viruses (Fraile et al. 2011) and basidiomycetes (Bruns et al. 2014; Thrall and Burdon 2003). These costs have the potential to prevent the resistance-breaking strain from completely dominating when a mixture of host cultivars are deployed. On a fully susceptible host, which can be infected by both wild-type and resistance-breaking pathogen strains, the costs of the resistancebreaking trait cause it to experience negative selective pressure. In the absence of immigration or new mutation, this would ultimately 
lead to loss of the resistance-breaking strain from the pathogen population. Such loss of unnecessary virulence is seen in field experimental evolution studies, where the lack of positive selection can result in a reduced frequency of individual resistance-breaking pathogen strains (Bousset et al. 2018). On the other hand, mutation to virulence can occur quite frequently, with wild-type viruses for example becoming resistance-breaking with as few as one or two nucleotide substitutions in the genes affecting avirulence (Harrison 2002). This can lead to resistance-breaking strains persisting at very low background frequencies in pathogen populations, even in the absence of selection from the corresponding resistant hosts (Stam and McDonald 2018).

As with any process that occurs over relatively long periods of time and over large spatial scales, mathematical modeling is a useful tool for examining the factors affecting resistance durability. In recent years, modeling in this area has integrated population genetics and epidemiology to consider durability largely in terms of the overall amount of host plant material infected in a given time period. van den Bosch and Gilligan (2003) introduced the number of additional uninfected host growth days as a measure of resistance durability, moving the focus away from simply looking at pathogen genotype frequencies as had often been done previously (Brown 1995; Lannou and Mundt 1996). The work by van den Bosch and Gilligan (2003) was further developed by Fabre et al. (2012), who represented the seasonally disturbed nature of the agricultural environment with a semidiscrete model (Mailleret and Lemesle 2009). In the Fabre et al. (2012) model, disease spread occurs in continuous time during cropping seasons but is reset upon harvesting, with dynamics in subsequent years being affected by a reservoir component which allows the pathogen to overwinter between discrete seasons (Burdon and Thrall 2008). Fabre et al. (2012) also implicitly characterized landscape spatial structure by modifying the relative contributions of within field, between field, and reservoir-driven infection. This characterization captures the overall degree of connectedness between different fields, along with the relative importance of ongoing primary infection from the reservoir component, but takes no account of explicit spatial effects such as dispersal distances or the scale or pattern of spatial heterogeneity. Results from the study by Fabre et al. (2012) highlighted the impact of resistance gene choice on durability, in terms of how easily it can be overcome by mutations in the pathogen avirulence gene, and with what associated fitness costs.

It is known from both theoretical and experimental studies that the mixing of cultivars with differing resistance properties can reduce the rate of disease spread in single and multipathogen strain systems (Jeger et al. 1981; Mundt 2002; Zhan and McDonald 2013). This can occur through dilution effects, where some of the force of infection from a given pathogen strain is wasted due to its reduced ability, or even inability, to infect the portions of the host population on which it is not specialized (Mundt 2002). It has been found in some theoretical model pathosystems that within field cultivar mixing is more effective at controlling disease spread than planting some fields wholly with susceptible plants and others with resistant plants (Skelsey et al. 2010). There are however frequent complications associated with the use of within field mixtures, including phenotypic differences between host varieties, such as in harvest dates, along with the perceived need for product purity (Burdon et al. 2016). These factors, which in part explain the limited use of mixtures in agriculture in the developed world, highlight the need to understand the effects on disease dynamics of employing host diversification at larger scales than within a single field, i.e., at the landscape or between field scale.

The number of studies looking at the effects of landscape scale host diversification combined with long range pathogen dispersal is limited, no doubt held back by the largely field scale nature of empirical work in this area (Plantegenest et al. 2007). This topic has received a degree of attention in theoretical studies however, with Skelsey et al. (2010) finding that the clustering of potato cultivation reduced the spread of late blight between clusters, but increased overall epidemic intensity due to higher spread within clusters. It has been posited that this scenario can potentially create a trade-off between within and between patch dispersal, resulting in maximized dispersal at intermediate scales of spatial clustering (Skelsey et al. 2013). Spatial structure is also relevant over longer timescales, with Papaix et al. (2013) showing, in a general theoretical metapopulation study, that spatial clustering of habitat patches facilitates specialization within a population, in addition to driving increased evolutionary speeds.

One component of landscape scale spatial structure that can potentially be optimized for disease control and resistance durability is the scale of spatial heterogeneity in the deployment of different host cultivars. The mechanism behind the efficacy of mixtures suggests that mixing host genotypes at smaller scales of spatial heterogeneity, and thereby creating smaller genotype unit areas, would benefit disease control by decreasing connectivity between patches of the same host cultivar (Mundt 2002). A modeling study by Papaïx et al. (2014), concerning a single pathogen strain, found similar benefits to mixing crop genotypes at smaller spatial scales when using major genes conferring complete resistance. However, these authors also found that for some levels of incomplete resistance, small patches of partially resistant crop could act as sinks for nearby pathogen populations in susceptible patches, and thereby act as stepping stones to increase overall disease incidence in the landscape with greater field mixing.

Some studies have looked more explicitly at resistance durability against multiple pathogen strains over evolutionary timescales. Sapoukhina et al. (2009) showed, using a reaction-diffusion model, that random mixtures in the host landscape provide greater long term disease suppression compared with patchy mixtures at larger scales of spatial heterogeneity. This is supported by a recent modeling study by Papaï et al. (2018), who showed that low levels of spatial aggregation, in a mixed landscape of fields planted with either susceptible or resistant cultivars, reduced epidemic intensities over both short term epidemiological timescales and at the long term evolutionary equilibrium. A field experimental evolution study by Bousset et al. (2018) provides a degree of empirical support for this general theory, with results suggesting that higher "genetic connectivity" within a host population facilitates higher levels of infection. Arguably, a potential weakness of this study was the implicit representation of genetic connectivity by greater experimental inoculation of host variety patches by their specialist pathogen strains, which means that it did not truly demonstrate a landscape scale spatial effect.

A number of recent theoretical studies have begun comparing and contrasting the various available strategies for the optimal deployment of resistance genes, such as using mosaics (between field spatial diversification), mixtures, rotations and pyramids (DjidjouDemasse et al. 2017; Fabre et al. 2015; Rimbaud et al. 2018). We believe however that the specific role of spatial diversification and the dynamics which affect this strategy are not currently well understood. While existing studies consistently point to smaller scales of spatial heterogeneity being optimal for durable and effective disease control in multistrain systems, there is generally little investigation of the factors that influence the strength of this spatial effect. In order for such spatial strategies to be employed in commercial agriculture, they will need to be cost effective, in that the benefit to resistance durability of planting fields of different cultivars at smaller scales of spatial heterogeneity must offset the likely increased financial cost and operational difficulty of farming in this manner. The principal aim of this study is therefore to examine the factors interacting with, and influencing the strength of, any such spatial effect. These factors include the dispersal characteristics of the pathogen, the fitness costs associated with the resistance-breaking trait, the efficacy of the host resistance gene, and the length of the timescale of interest. 


\section{MATERIALS AND METHODS}

We extend the model used by Fabre et al. (2012) to explicitly include spatial structure and different pathogen strains. Our SI (susceptible, infected) model tracks two pathogen strains, a wildtype ( $w t)$ and a resistance-breaking $(r b)$ genotype (the principal variables and parameters used in this model are summarized in Table 1). The underlying host landscape consists of a number $\left(n_{f}=\right.$ 100) of cultivated fields, each with a constant number of plants/ plant tissue units $\left(n_{p}=1,000\right)$ of either a susceptible $(S)$ or resistant $(R)$ cultivar type. We use "plants" throughout for consistency with the terminology of Fabre et al. (2012), although for agricultural fields our quantum would of course correspond to a possibly very large number of individual plants $(1 / 1,000$ th of the total number of plants contained in a given field). Note that the use of continuous state variables combined with the method of epidemic parametrization (Supporting Notes S1) makes the results ultimately independent of the number of plants/plant tissue units $\left(n_{p}\right)$. The proportion of resistant fields (the cropping ratio) is set evenly with that of susceptible fields at $\phi=0.5$.

Resistance acts in a gene-for-gene system where the $w t$ strain can freely infect $S$ fields, but has reduced fitness (that may be zero) when infecting $R$ fields. The $r b$ strain has equal infective ability on both host genotypes, but may have a reproductive fitness cost $(\delta)$ (expressed on both host genotypes) associated with its resistancebreaking trait. The focus of our model on disease spread at the landscape scale, with kernel driven pathogen dispersal, makes it appropriate for application to any gene-for-gene crop disease system with a foliar wind dispersed pathogen (such as many rusts or powdery mildews). The arbitrary nature of the spatial scales in the model mean that it could also be applied to smaller spatial scales such as within field mixtures. In this case, the host fields described in this model could instead represent smaller subunits of hosts within fields or even individual plants.

The overall number of infected plants in each field is simulated for $n_{d}=120$ days over $n_{y}$ seasons in a semidiscrete modeling approach. The choice of 120 growing days per season is arbitrary since the method of epidemic parametrization (Supporting Notes S1) makes the results ultimately independent of $n_{d}$. In the semidiscrete model, continuous time dynamics in ordinary differential equations (ODEs) are used for the within season component, and discrete dynamics are used for the pathogen infecting and overwintering in the reservoirs (Mailleret and Lemesle 2009). The reservoir components are localized to each field, and represent plants found in field margins and hedgerows, as well as crop volunteers, that act as alternative hosts for the pathogen. In all simulations, $I_{w t, x, y}(0)=I_{r b, x, y(0)}=0$ (i.e., the number of infected plants within fields is set to zero at the beginning of each season), with all epidemics started by primary infection from the reservoir of inoculum.

The total area under the disease progress curve (AUDPC) over the $n_{y}$ seasons is used as the measure of epidemic intensity (Madden et al. 2007). As a measure of resistance durability this is equivalent to the number of uninfected host growth days used by van den Bosch and Gilligan (2003). The AUDPC is normalized to a value between 0 and 1 to obtain the average proportion of plants infected across the sequence of epidemic seasons (epidemic intensity $=\operatorname{AUDPC} /\left(n_{f} n_{p} n_{y} n_{d}\right)$ ). Healthy plants can become infected through three alternative routes: from infected plants in the same field at rate $\beta_{F}$, from infected plants in other fields at rate $\beta_{C}$, and from the reservoir of inoculum at rates $\alpha_{w t, y}$ or $\alpha_{r b, y}$ in season $y$ for the $w t$ and $r b$ pathogen strains, respectively. The pathogen population size in the reservoir is not explicitly modeled but is represented by these rate parameters $\left(\alpha_{w t, y}\right.$ and $\left.\alpha_{r b, y}\right)$ by scaling the baseline rate of infection from the reservoir component $\alpha_{E}$.

The values of the transmission parameters $\beta_{F}, \beta_{C}$, and $\alpha_{E}$ were optimized by following Fabre et al. (2012) to set the overall intensity of, and the relative contributions of the three infection routes to, a baseline epidemic scenario (Supporting Notes S1). In a nonspatially explicit setting, the general effects of changing these parameters on resistance durability have been explored by Fabre et al. (2012). The version of the model used for this optimization uses a landscape with susceptible fields only $(\phi=0)$. The values of the transmission parameters are such that in this case the three transmission routes have an equal contribution to maintaining the epidemic, and the mean proportion of plants infected during a season is 0.5 . The rational for this is that such a baseline epidemic, with balanced routes of infection and a moderate epidemic intensity, will be clearly responsive to changes in any of the other parameters we explore in this model. This is not intended to accurately represent a real life landscape scale pathosystem, but instead ensures that any patterns found in the results can be more easily analyzed.

The fields are located within a square landscape of $10 \times 10$ arbitrary distance units, where the distances $(d)$ between each pairwise combination of fields are calculated and used in a normalized negative exponential dispersal kernel (of the form $K=\frac{\eta^{2}}{2 \pi} e^{-\eta d}$ with mean dispersal distance $1 / \eta$ ) to calculate each

TABLE 1. Parameters and variables used in the mathematical model

\begin{tabular}{|c|c|c|}
\hline Symbol & Parameter/variable description & Constraints/values \\
\hline$I_{w t, x, y}$ & Number of plants in field $x$ and season $y$ infected by the $w t$ strain & \\
\hline$I_{r b, x, y}$ & Number of plants in field $x$ and season $y$ infected by the $r b$ strain & \\
\hline$n_{f}$ & Number of fields & 100 \\
\hline$n_{p}$ & Number of plants/plant tissue units per field & 1,000 \\
\hline$\phi$ & Cropping ratio (proportion of resistant fields) & 0.5 \\
\hline$n_{d}$ & Number of days in a season & 120 \\
\hline$n_{y}$ & Number of seasons & $1 \leq n_{y} \leq 80$ \\
\hline$\theta$ & Background equilibrium frequency of $r b$ strain on $S$ host & 0.01 \\
\hline$\lambda$ & Degree of "instability" in the reservoir host pathogen population & $0.5(\lambda \in[0,1])$ \\
\hline$\delta$ & Fitness cost of the $r b$ trait & $0 \leq \delta \leq 1$ \\
\hline$\gamma$ & Susceptibility of the $R$ host to the $w t$ strain & $0 \leq \gamma \leq 1$ \\
\hline$K[z, x]$ & 2D normalized dispersal kernel coupling field $z$ to field $x$ & $z \neq x, K=\frac{\eta^{2}}{2 \pi} e^{-\eta d}$ \\
\hline$\eta$ & Dispersal kernel parameter & 3 levels: $1,2,3$ \\
\hline$A_{0}$ & Baseline AUDPC for one season in a fully susceptible landscape & $A_{0}=\sum_{x=1}^{n_{f}}\left(\int_{0}^{n_{d}} I_{S, x, y}(t) \mathrm{d} t\right)$ \\
\hline$\beta_{F}$ & Within field infection rate & (see text and SI) \\
\hline$\beta_{C}$ & Between field infection rate & (see text and SI) \\
\hline$\alpha_{E}$ & Baseline rate of infection from the reservoir & (see text and SI) \\
\hline$\alpha_{w t, y}$ & Rate of $w t$ infection from the reservoir & \\
\hline$\alpha_{r b, y}$ & Rate of $r b$ infection from the reservoir & \\
\hline$A_{w t, x, y-1}$ & AUDPC for the $w t$ epidemic in field $x$ in the previous season & $\int_{0}^{n_{d}} I_{w t, x, y-1}(t) \mathrm{d} t$ \\
\hline$A_{r b, x, y-1}$ & AUDPC for the $r b$ epidemic in field $x$ in the previous season & $\int_{0}^{n_{d}} I_{r b, x, y-1}(t) \mathrm{d} t$ \\
\hline
\end{tabular}


pairwise strength of spatial coupling. This form of kernel is commonly used to represent dispersal in epidemiological systems where relatively short ranged dispersal is of primary importance (Parnell et al. 2009, 2010, 2015). Although our model could represent a landscape scale epidemic, the vast majority of dispersal still occurs over relatively short distances. The importance of rare long distance dispersal events is limited due to the endemic disease scenario with both strains of pathogen already present in all spatial locations, albeit with the resistance-breaking strain at a low initial density. Each simulation was repeated 100 times, with different random field locations in each replicate.

To explore the effect of the scale of spatial heterogeneity in the arrangement of $S$ and $R$ fields on epidemic intensities, the landscape area is arranged (with cropping ratio $\phi=0.5$ ) into a number of patches. Each patch only contains fields of a single host genotype ( $S$ or $R$ ). At the largest scale, the landscape is split in half, with all the $S$ fields in one half and all the $R$ fields in the other. At the smallest scale, the landscape is split into 64 patches with alternating host genotypes. Four intermediate scales are also used, for a total of six landscape templates with different patch sizes and scales of spatial heterogeneity (Table 2). We characterize the scale of spatial heterogeneity via the interior edge/area (E/A) ratio of each landscape (Supporting Notes S2). This metric captures the degree of contact between the two different host patch types, relative to the overall size of the landscape. It serves as an effective proxy for the overall proximity of the two host field types to each other, and can

TABLE 2. The edge/area (E/A) ratios of the landscapes at each of the six scales of spatial heterogeneity (Fig. 1 and Supporting Notes S2)

\begin{tabular}{lc}
\hline Number of patches & E/A ratio \\
\hline 2 & $10 / 10^{2}=0.1$ \\
4 & $20 / 10^{2}=0.2$ \\
8 & $40 / 10^{2}=0.4$ \\
16 & $60 / 10^{2}=0.6$ \\
32 & $100 / 10^{2}=1$ \\
64 & $140 / 10^{2}=1.4$ \\
\hline
\end{tabular}

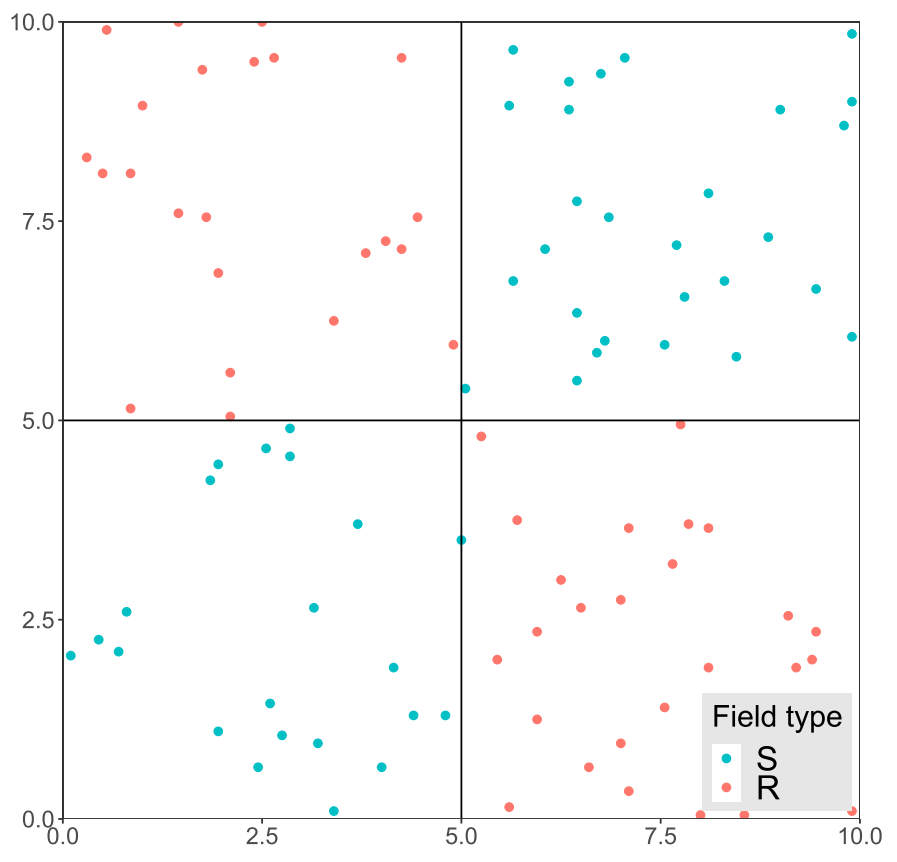

4 patches, Edge/Area ratio $=0.2$ also be applied to landscapes with irregular patch shapes, variable patch sizes, and different total areas. The pattern of host genotype patches is used as a template to place the two types of field, using random coordinates, in alternating patches within the landscape (Fig. 1).

The spread of the two pathogen strains in a given field within a season follows the general pseudo-equation form:

$$
\begin{aligned}
& \underset{\text { Rate of change of }}{\text { infected plants }}=\left(\begin{array}{c}
\text { Uninfected } \\
\text { plants }
\end{array}\right) \times \\
& \left(\begin{array}{ccc}
\text { Rate of } & \text { Rate of } & \text { Rate of } \\
\text { primary (reservoir }) & + \text { between field } \\
\text { infection } & \text { infection } & \text { ithin field } \\
\text { infection }
\end{array}\right)
\end{aligned}
$$

and, defining $S_{x, y}=n_{p}-I_{w t, x, y}-I_{r b, x, y}$ for notational convenience, is described by the deterministic ODE system:

$$
\begin{aligned}
& \frac{\mathrm{d} I_{w t, x, y}}{\mathrm{~d} t}=\gamma^{*}\left(S_{x, y}\right)\left(\alpha_{w t, x, y}+\beta_{C}\left(\sum_{z=1}^{n_{f}} I_{w t, z, y} K[z, x]\right)+\beta_{F} I_{w t, x, y}\right) \\
& \frac{\mathrm{d} I_{r b, x, y}}{\mathrm{~d} t}=(1-\delta)\left(S_{x, y}\right)\left(\alpha_{r b, x, y}+\beta_{C}\left(\sum_{z=1}^{n_{f}} I_{r b, z, y} K[z, x]\right)+\beta_{F} I_{r b, x, y}\right)
\end{aligned}
$$

in which

$$
\gamma^{*}=\left\{\begin{array}{l}
1 \text { if } x \text { is of type } S \\
\gamma \text { if } x \text { is of type } R
\end{array}\right.
$$

in equation 2 .

In equations 2 and $3, x$ indicates variables pertaining to a particular field, $K[z, x]$ is the dispersal kernel coupling field $z$ to field

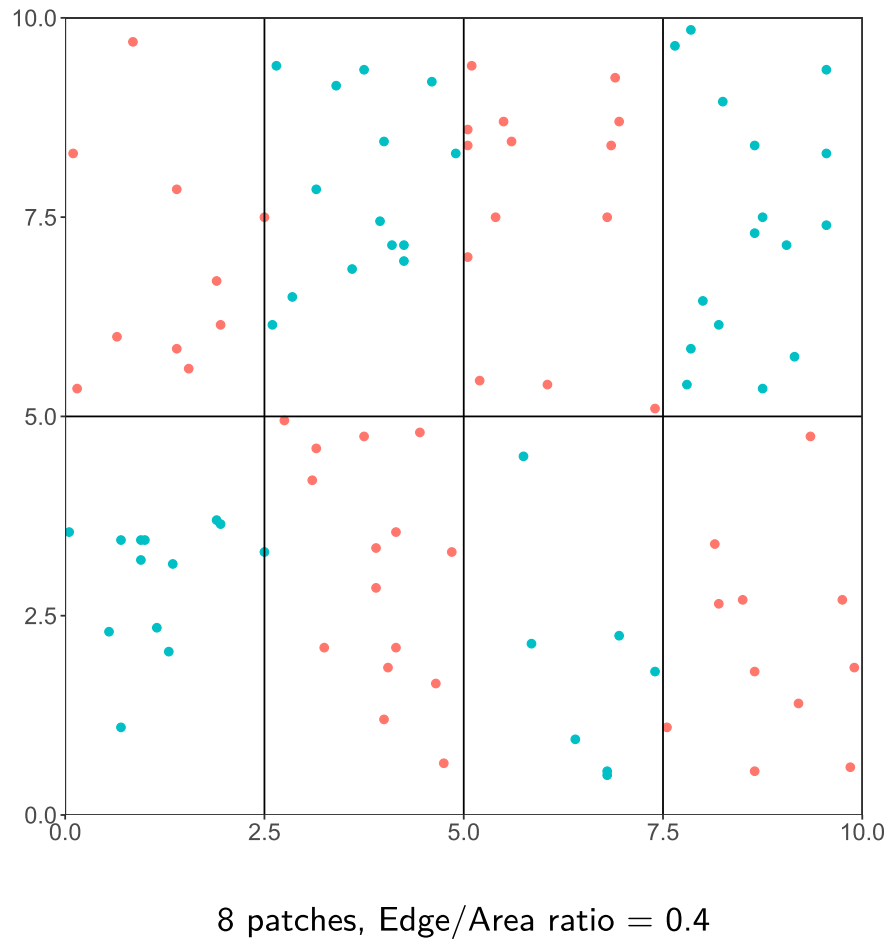

Fig. 1. Example random field arrangements for two patch templates within the agricultural landscape. Each dot within the landscapes represents a single field containing plants of a given cultivar. 
$x$, and $z \neq x$. Here $I_{w t, x, y}$ is the number of plants infected by the $w t$ strain while $I_{r b, x, y}$ is the number infected by the $r b$ strain (both being in field $x$ and in season $y$ ). The cost of the resistance-breaking trait is $\delta$, which is assumed act on the rate of sporulation on both the primary and reservoir host, while $\gamma$ is the susceptibility of the $R$ host to the $w t$ strain. If resistance to the $w t$ strain is complete $\gamma=0$, meaning that the $w t$ epidemic in an $R$ field (equation 2 where $\gamma^{*}=\gamma$ ) does not occur.

The terms $\alpha_{w t, x, y}$ and $\alpha_{r b, x, y}$ represent the specific infection rates of each pathogen genotype from the local reservoir, where the reservoir host is assumed to be selectively neutral to both pathogen genotypes. These rates are subject to change between seasons from initial values of $\alpha_{w t, x, 0}=\alpha_{E}$ and $\alpha_{r b, x, 0}=\theta \alpha_{E}$, where $\theta$ is the initial frequency of the $r b$ strain. This initial frequency of infection could potentially originate from the reservoir host, and is assumed to not depend on the fitness cost of the $r b$ trait $(\delta)$. The contributions of the reservoir components to the epidemic in season $y$ are given by

$$
\begin{aligned}
& \alpha_{w t, x, y}=\lambda \frac{\alpha_{E}\left(A_{w t, x, y-1}\right)}{A_{0} / n_{f}}+(1-\lambda) \alpha_{w t, x, y-1} \\
& \alpha_{r b, x, y}=\lambda \frac{\alpha_{E}\left(A_{r b, x, y-1}\right)}{A_{0} / n_{f}}+(1-\lambda) \alpha_{r b, x, y-1}
\end{aligned}
$$

Here, $A_{w t, x, y^{-1}}=\int_{0}^{n_{d}} I_{w t, x, y^{-1}}(t) \mathrm{d} t$ and $A_{r b, x, y^{-1}}=\int_{0}^{n_{d}} I_{r b, x, y^{-1}}(t) \mathrm{d} t$, the AUDPCs for the epidemics in the previous season caused by the $w t$ and $r b$ pathogen genotypes, respectively, in a given individual field $x$. The baseline landscape AUDPC $A_{0}=\sum_{x=1}^{n_{f}}\left(\int_{0}^{n_{d}} I_{S, x, y}(t) \mathrm{d} t\right)$, calculated from one season in the fully susceptible baseline model, is used to scale the previous season's epidemic to measure the proportional reduction in epidemic intensity due to the presence of $R$ fields.

The parameter $\lambda \in[0,1]$ characterizes the reservoir. High values of $\lambda$ indicate a rapidly changing reservoir with primary infection largely depending on the intensity of the previous season's field epidemics. This scenario could potentially be caused by a short reservoir host lifespan, a high rate of spread within the reservoir, or a small reservoir size. Low values of $\lambda$ indicate a more stable reservoir, with a larger effect of older epidemics damping changes to $\alpha_{w t, x, y}$ and $\alpha_{r b, x, y}$. A value of $\lambda=0.5$, equally weighting both terms in equations 5 and 6 , is used here.

\section{RESULTS}

An illustrative time series of seasonal disease progress curves for an example landscape with a high E/A ratio is shown in Figure 2. In this figure, the epidemic intensity of the $r b$ strain on the $S$ host increases above that of the $w t$ on the same host. This is due to the small scale of spatial heterogeneity allowing the $r b$ strain to partially outcompete the $w t$, and does not indicate an oscillation in the frequencies of the two strains on this host. The average seasonal epidemic intensity (average proportion of plants infected throughout the epidemic) over a 40 season time period invariably decreases as the E/A ratio of the landscape is increased (Fig. 3). This is due to the larger dilution caused by greater mixing of the two host genotype field types at smaller scales of spatial heterogeneity. Epidemic intensity is initially measured here over a 40 season time period, to balance short term and long term evolutionary dynamics, although the effect of varying the time frame of interest is described in the effect of timescale section. The reduction in epidemic intensities due to the planting of $S$ and $R$ fields at smaller scales of spatial heterogeneity within the landscape (higher E/A ratios) is referred to as the "spatial suppressive effect" throughout the remainder of this article.

The $w t$ epidemic is suppressed at higher E/A ratios (Fig. 3) due to the smaller spatial grain causing an increase in the proportion of dispersed $w t$ inoculum from $S$ fields that is wasted as it lands on, but is unable to infect, $R$ fields. The $r b$ epidemic in $R$ fields meanwhile is suppressed by a corresponding process in which there is an increase in the proportion of $r b$ inoculum that lands on $S$ fields. The $r b$ inoculum is in direct competition with $w t$ inoculum for uninfected host tissue within $S$ fields, resulting in a lower intensity $r b$ epidemic than would take place in an $R$ field. The consequent $r b$ genotype dispersal from these $S$ fields back onto the nearby $R$ fields therefore has a lower force of infection than in $R$ field to $R$ field transmission over the same distance. The reduction in the intensity of the $r b$ epidemic on $R$ fields is compensated to a certain extent by the increase in the frequency of the $r b$ genotype on $S$ fields as the two field genotypes are more closely mixed together in space. Whether this compensation ultimately increases or decreases the intensity of the overall landscape $r b$ epidemic, as the E/A ratio is increased, depends upon the genetic parameters (Fig. 4).

Effect of the kernel parameter $\boldsymbol{\eta}$. The gradient of the spatial suppressive effect (the rate of change in the response of average seasonal epidemic intensity to $\mathrm{E} / \mathrm{A}$ ratio) decreases as the $\mathrm{E} / \mathrm{A}$ ratio

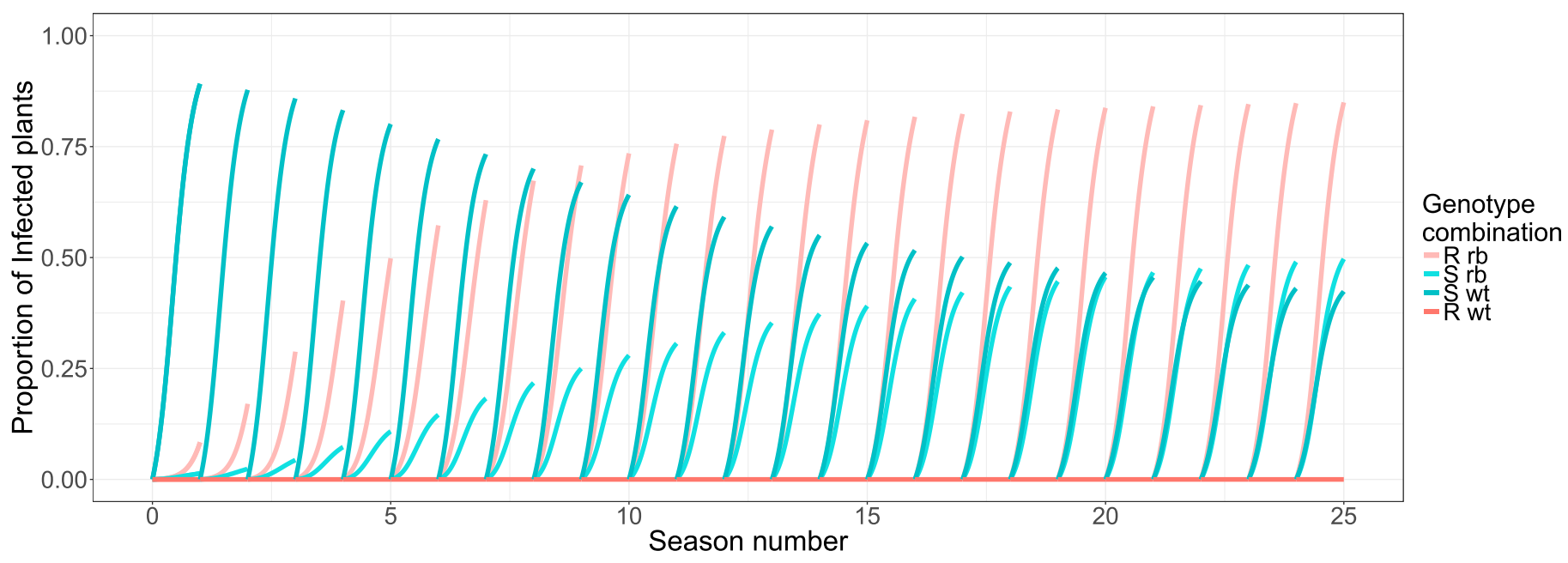

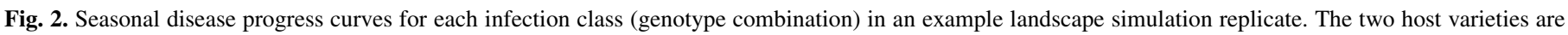

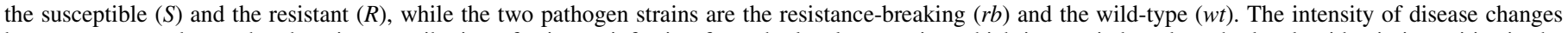

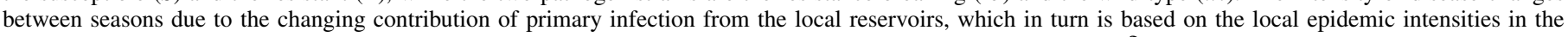

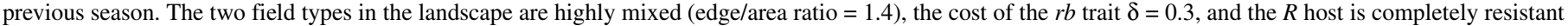
to the $w t$ strain $\gamma=0$. Only 25 seasons are shown here so that the individual disease progress curves can be clearly seen. 
is increased (Fig. 3). For a higher mean dispersal distance, the rate of change in epidemic intensity is quite steep at low E/A ratios, but then almost completely flattens out at high E/A ratios. In contrast, for a lower mean dispersal distance, there is comparatively little change in the gradient of the spatial suppressive effect as the E/A ratio is increased. This implies that higher mean dispersal distances cause the strength of the spatial suppressive effect to be washed out at small scales of spatial heterogeneity. This occurs because the high mean dispersal distance of the pathogen limits the impact of any further decrease in the scale of spatial heterogeneity. In general, this means that in the reverse direction, as the mean dispersal distance decreases, the spatial suppressive effect is relevant over a larger range of E/A ratios. Despite these differences, the results shown in Figure 3 are somewhat similar to each other in their overall patterns due to the relatively similar shapes of the relevant dispersal kernels.
Effect of the cost of the $\boldsymbol{r} \boldsymbol{b}$ trait $\delta$. The overall size of the spatial suppressive effect increases as the cost of the $r b$ trait $\delta$ is increased from 0 to 0.4 (Fig. 4). Furthermore, the extent to which the pathogen genotype frequencies on the $S$ host change, as we move from larger to smaller scales of spatial heterogeneity, also depends on the cost of the $r b$ trait. When $\delta=0$ (Fig. 4A), the $r b$ genotype is able to take advantage of the greater proportion of host fields that it can infect, and the close proximity of the two field types at smaller spatial scales of heterogeneity, allowing it to outcompete the $w t$ genotype. This replacement of pathogen genotypes at different E/A ratios is seen to a lesser extent when $\delta=0.2$ (Fig. 4B), and is almost absent when $\delta=0.4$ (Fig. 4C). As the fitness cost $\delta$ increases, the $r b$ genotype is unable to compete as effectively with the $w t$ genotype on the $S$ host at small scales of spatial heterogeneity. This is despite the close proximity of large numbers of $R$ fields, which act as a major source of $r b$ inoculum, to the $S$ fields.

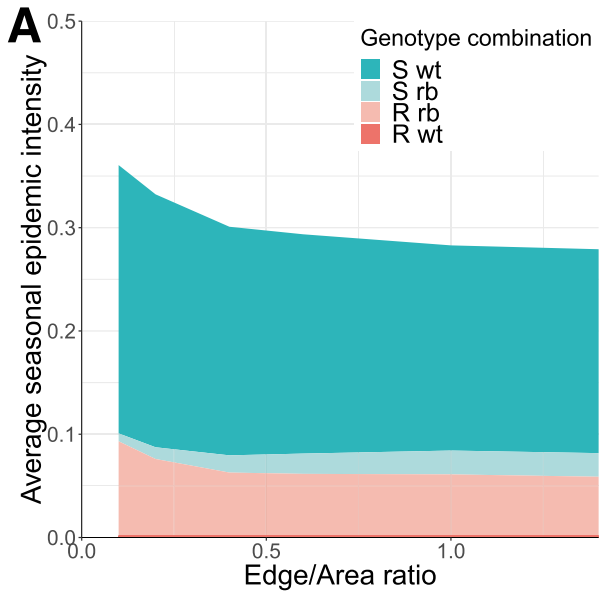

$$
\begin{gathered}
\eta=1 ; \text { higher mean dispersal } \\
\text { distance }
\end{gathered}
$$

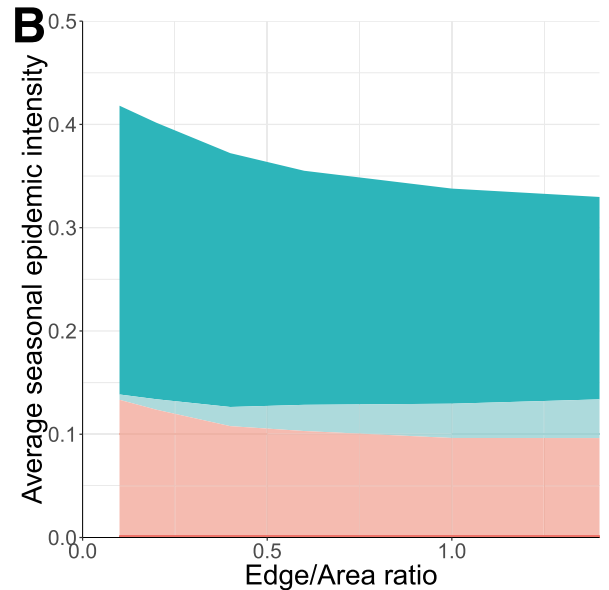

$\eta=2$

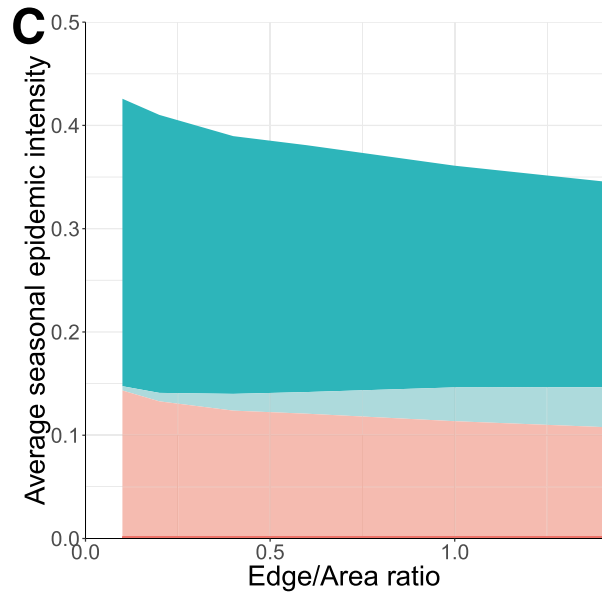

$\eta=3$; lower mean dispersal

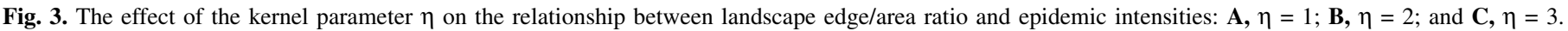

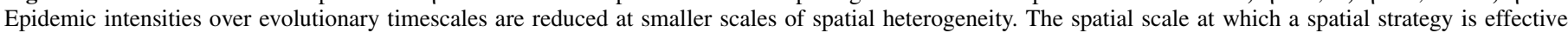

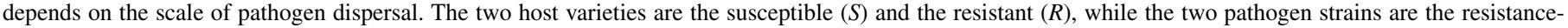

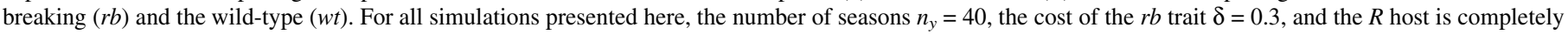
resistant to the $w t$ strain $\gamma=0$.

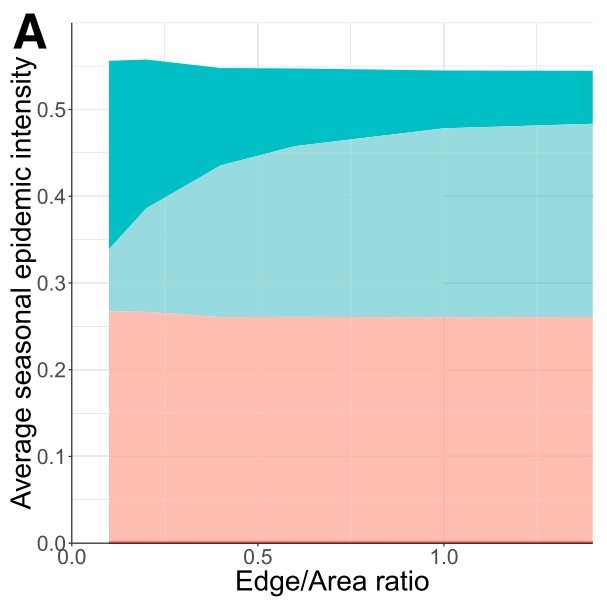

$$
\delta=0
$$

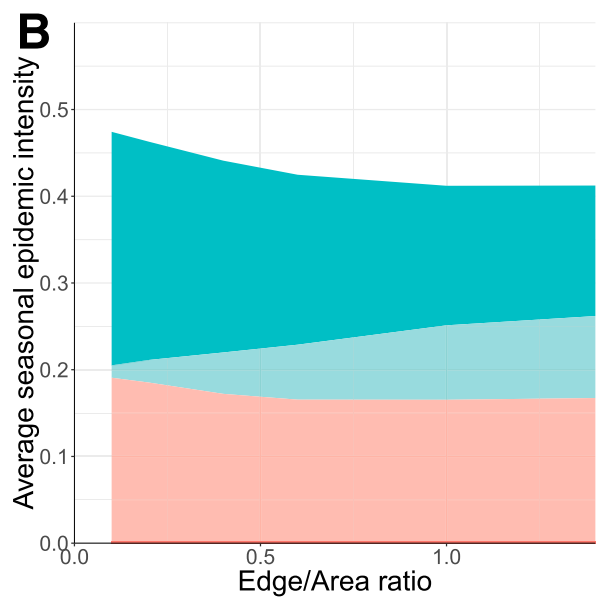

$\delta=0.2$

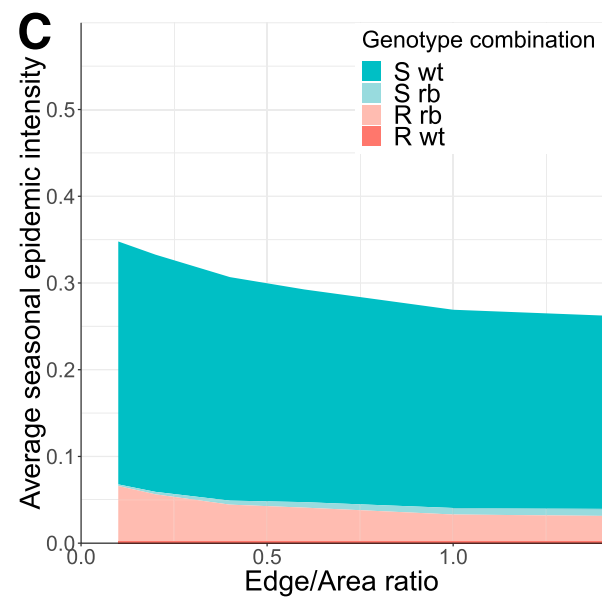

$\delta=0.4$

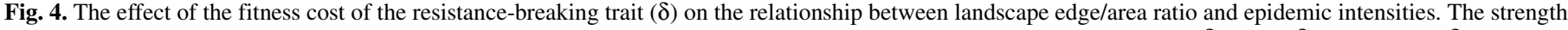

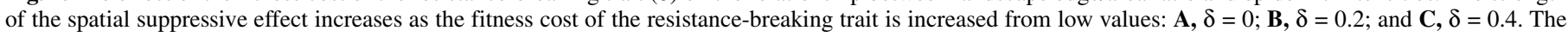

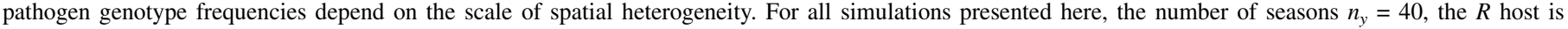
completely resistant to the $w t$ strain $\gamma=0$, and the kernel parameter $\eta=2$. 
Plotting overall epidemic intensity against the full range of values for the fitness cost of the $r b$ trait $(\delta)$, at both the low and high ends of the $\mathrm{E} / \mathrm{A}$ ratio scale (i.e., for $\mathrm{E} / \mathrm{A}=0.1$ and 1.4 ), allows these contrasting spatial scenarios to be compared (Fig. 5). For both E/A ratios, epidemic intensities decrease as $\delta$ is increased, up to $\delta=0.6$ where the $r b$ trait is too expensive for that pathogen genotype to invade and there is no further effect of increasing $\delta$ (Fig. 5A). The strength of the spatial suppressive effect can be ascertained by plotting the difference between the epidemic intensities for the two E/A ratio values (Fig. 5B). The variability in this effect was estimated by randomly pairing together, and taking the difference between, individual simulation results from the two groups of E/A ratio values. The strength of the spatial suppressive effect increases from $\delta=0$ to 0.3 , but decreases from $\delta=0.4$ to 0.6 . A small spatial suppressive effect is still seen at $\delta=0$ due to the small scale of spatial heterogeneity disrupting the transient $w t$ epidemic, before the $w t$ strain is outcompeted by the $r b$ and reaches its near zero evolutionary equilibrium frequency.

The initial increase in the strength of the spatial suppressive effect is due to a steeper gradient of change, in the response of overall epidemic intensity to changes in $\delta$, at small scales of spatial heterogeneity $(\mathrm{E} / \mathrm{A}$ ratio $=1.4)($ Fig. $5 \mathrm{~A})$. This steeper change with $\delta$ is in turn caused by rapidly changing $r b$ dynamics on the $S$ host, which are a larger driver of system sensitivity with greater field mixing (Supporting Fig. S2). Here, any increase in $\delta$ reduces the competitive ability of the $r b$ genotype against the $w t$ on susceptible hosts, which consequentially increases the amount of $r b$ inoculum that is "wasted" as it disperses onto these $S$ hosts, thereby increasing the spatial suppressive effect strength.

The subsequent fall in the strength of the spatial suppressive effect is correspondingly due to a steeper gradient of change with $\delta$ at large scales of spatial heterogeneity $(\mathrm{E} / \mathrm{A}$ ratio $=0.1)($ Fig. $5 \mathrm{~A})$. In this range of $\delta$ values there is an increased sensitivity to changes in $\delta$ of the $r b$ epidemic on the $R$ host, relative to that on the $S$ host (Supporting Fig. S2). This is primarily because the $r b$ genotype is already unable to compete effectively with the $w t$ on the susceptible host in this range, and therefore does not respond to further changes in the fitness cost. These rapidly changing $R r b$ dynamics are a larger driver of system sensitivity with less field mixing.

The value of $\delta$ for which the gradient of overall epidemic intensity change with $\delta$ is equal at both small and large scales of spatial heterogeneity ( $\delta=0.3$ to 0.4 ) is the point of maximum spatial suppressive effect on epidemic intensities (i.e., the maximum distance between the curves). At this point, the combined sensitivity effects, of $r b$ dynamics on both $S$ and $R$ hosts to changes in $\delta$, have the same net result at high and low landscape E/A ratios.

Partial resistance. Here, we relax the assumption that the $w t$ strain cannot infect resistant hosts (Fig. 6). As $\gamma$ is increased above 0 , the $w t$ genotype becomes able to infect the $R$ host, and at higher frequencies with smaller scales of spatial heterogeneity (high E/A ratios) (Fig. 6A and B). The reduced efficacy of the resistance gene allows the $w t$ strain to compete more effectively with the $r b$ strain on the $R$ host, particularly at high E/A ratios where the field types are more greatly mixed in space. The strength of the spatial suppressive effect, from using a high rather than a low E/A ratio, is shown in Figure 6D. For an intermediate cost of the $r b$ trait $(\delta=0.3)$, the strength of the spatial suppressive effect decreases to zero as $\gamma$ is increased from 0 to 0.6 . This effect is due to a reduction in the proportion of $w t$ inoculum that is wasted in its increased dispersal onto $R$ fields at smaller scales of spatial heterogeneity. When $\gamma=1$ there is no effect of the scale of spatial heterogeneity, as the landscape is then made up of entirely susceptible hosts, and therefore the $w t$ genotype is able to fully outcompete the $r b$ genotype.

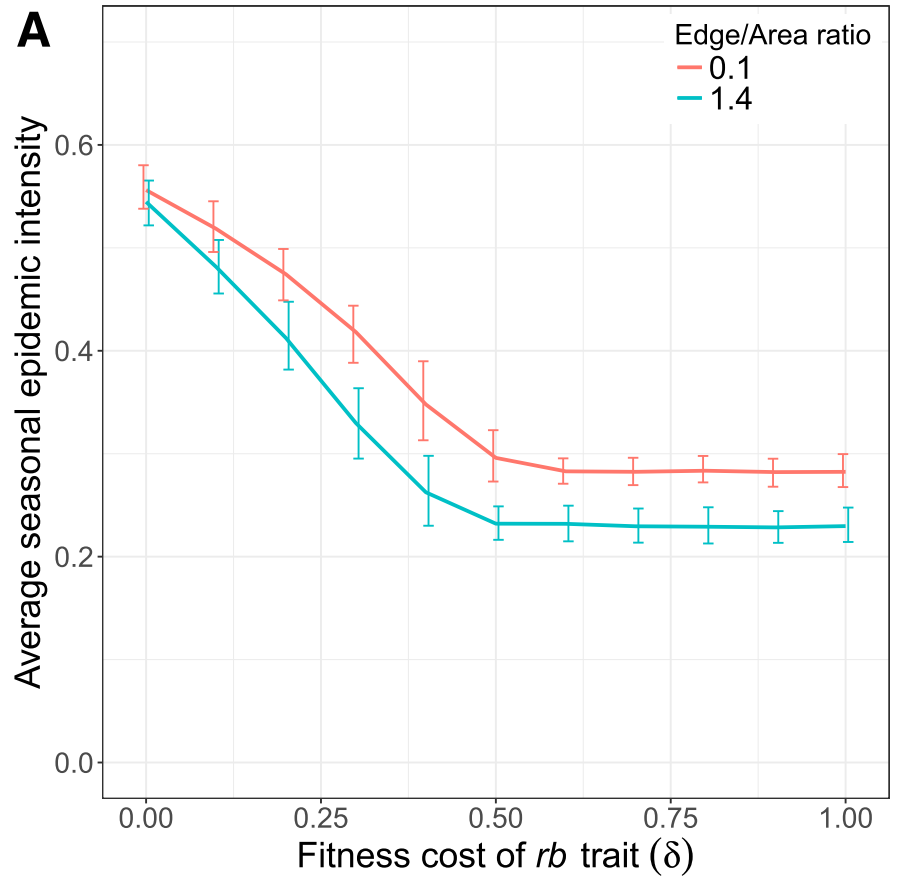

Epidemic intensities

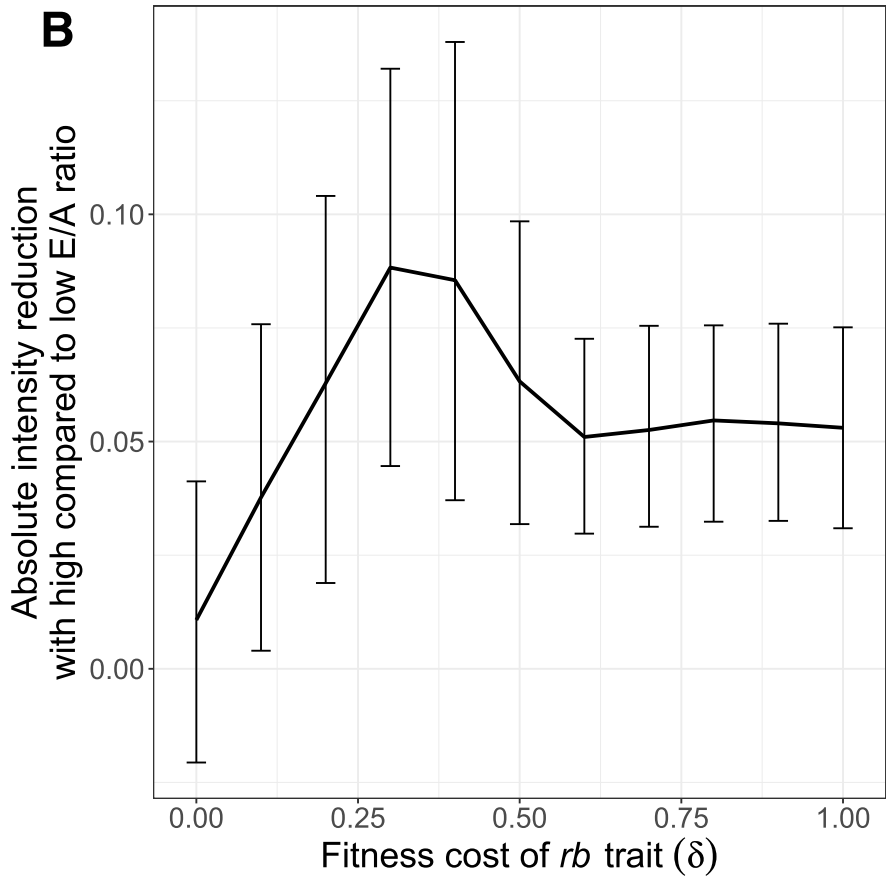

Absolute differences

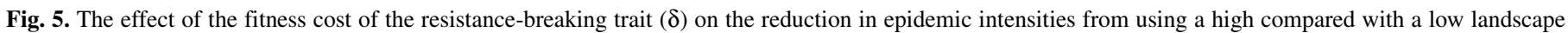

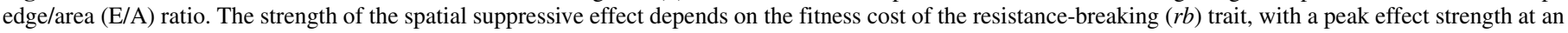

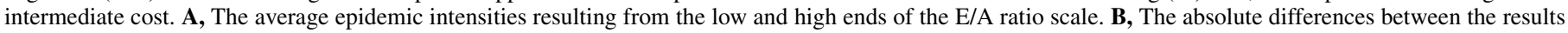

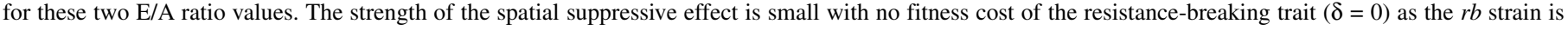

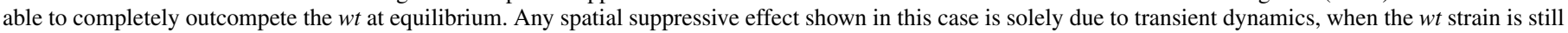

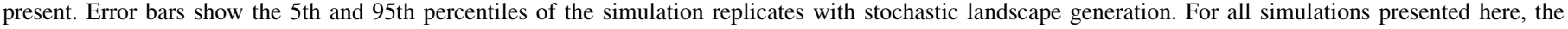
number of seasons $n_{y}=40$, the $R$ host is completely resistant to the $w t$ strain $\gamma=0$, and the kernel parameter $\eta=2$. 
There is a range of $\gamma$ values, from 0.6 to 0.9 for $\delta=0.3$ (Fig. 6D), for which the strength of the spatial suppressive effect dips below zero and becomes negative, indicating that smaller scales of spatial heterogeneity increase epidemic intensities. This increase only occurs at $\delta$ and $\gamma$ combinations where the $r b$ strain is not fit enough to invade, or is only present at extremely low frequencies (Fig. 7). In addition to this, the increased epidemic intensities are only observed at medium to high values of $\gamma$, and are most apparent at medium $\gamma$ values. This effect is due to the inability of the wt pathogen genotype to sustain epidemics in the $R$ host fields without the presence of nearby $S$ fields to act as a source of $w t$ inoculum. At higher E/A ratios however, where the field types are mixed at smaller scales of spatial heterogeneity, the closer proximity of $S$ field $w t$ sources enables greater infection of the $R$ field sinks, thereby increasing the overall proportion of infected plants in the landscape, despite the reduction in epidemic intensities on the $S$ fields themselves. At very high values of $\gamma$, the $w t$ genotype is fit enough to better sustain epidemics at large scales of spatial heterogeneity (low E/A ratios), so the negative spatial suppressive effect is reduced in strength (and cannot occur at all with $\gamma=1$ ). At low values of $\gamma$, spatial suppression of epidemic intensities are still observed, despite the noninvasion of the $r b$ strain, due to the very low fitness of the $w t$ genotype on the $R$ host, which severely limits $w t$ epidemics in $R$ fields at any scale of spatial heterogeneity.

Effect of timescale. By relaxing the assumption of a 40 season time period, we can measure the strength of the spatial suppressive effect, or the difference in epidemic intensities between low and high landscape E/A ratios, across a wide range of eco-evolutionary timescales. The average seasonal epidemic intensity generally increases as the number of seasons is increased (Fig. 8A, B, and C). This is due to the increasing frequency of the $r b$ genotype, which facilitates greater infection of $R$ fields as the system approaches its long term evolutionary equilibrium. In the case where the fitness cost of the $r b$ trait $\delta=0$, there is no significant spatial suppressive effect on epidemic intensities over 80 seasons (i.e., the red and blue curves converge in Figure 8A). Here, the $r b$ strain is able to completely outcompete the $w t$ on both host genotypes at the long term evolutionary equilibrium, meaning that the scale of spatial heterogeneity has no effect. A transient spatial suppressive effect does however occur over a low to medium number of seasons, as the system has not yet reached its equilibrium state and the $w t$ strain is still present at significant frequencies.

In some cases there is a specific number of seasons, for a given fitness cost of the $r b$ trait $(\delta)$, over which the greatest spatial suppressive effect on epidemic intensities can be achieved (Fig. 8D, $\mathrm{E}$, and F). For $\delta=0$ and $\delta=0.2$ (Fig. 8D and E), this peak effect strength occurs over a relatively short time period, approximately 4 and 11 seasons, respectively. With $\delta=0.4$ however, the greatest spatial suppressive effect is observed as the system approaches its long term evolutionary equilibrium.

The initial increase in the strength of the spatial suppressive effect, from the first season to the intermediate peak, is due to a faster increase in epidemic intensities at a low E/A ratio compared with a high E/A ratio. This is because the newly emergent $r b$ genotype is able to propagate rapidly in the highly aggregated $R$ fields at low E/A ratios. The $r b$ genotype initially spreads less quickly in $S$ fields, and therefore at high E/A ratios, due to the high level of competition with the coexisting $w t$ pathogen genotype. Beyond the peak in spatial suppression, if it is present, the strength of the spatial suppressive effect declines as epidemic intensities begin to increase faster at high compared with low E/A ratios. This is due to the increased importance of the $r b$ strain spread on $S$ hosts. The overall $r b$ frequency in the landscape is higher, than it is over a lower number of seasons, resulting in a faster growth rate on the $S$ host as it competes more effectively with the $w t$ strain. The $r b$ spread on the $R$ host with a low E/A ratio, however, is already well advanced at this stage, causing its rate of spread to decline.

The two key components here are the rates of spread of the $r b$ pathogen strain on each of the two varieties of host. The scale of spatial heterogeneity determines how impactful each of these components is as the number of seasons is varied. The peak in the strength of spatial suppression occurs where the combined impact of the two processes is the same at both small and large scales of spatial heterogeneity. This peak occurs over a higher number of

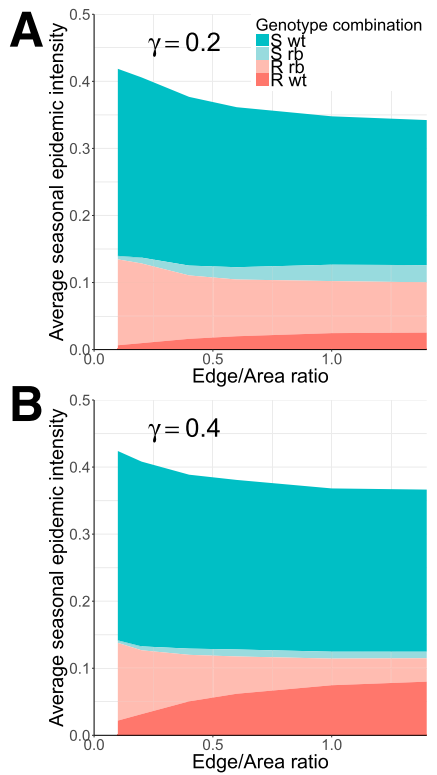

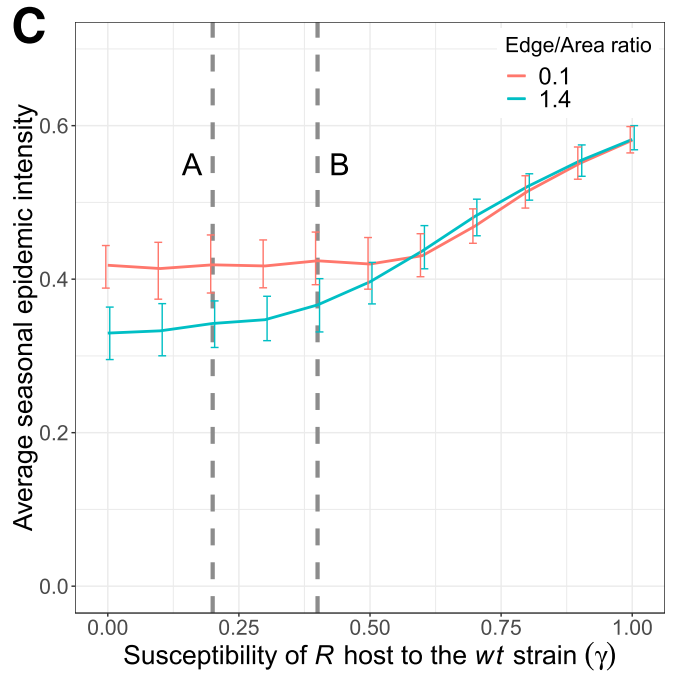

Epidemic intensities

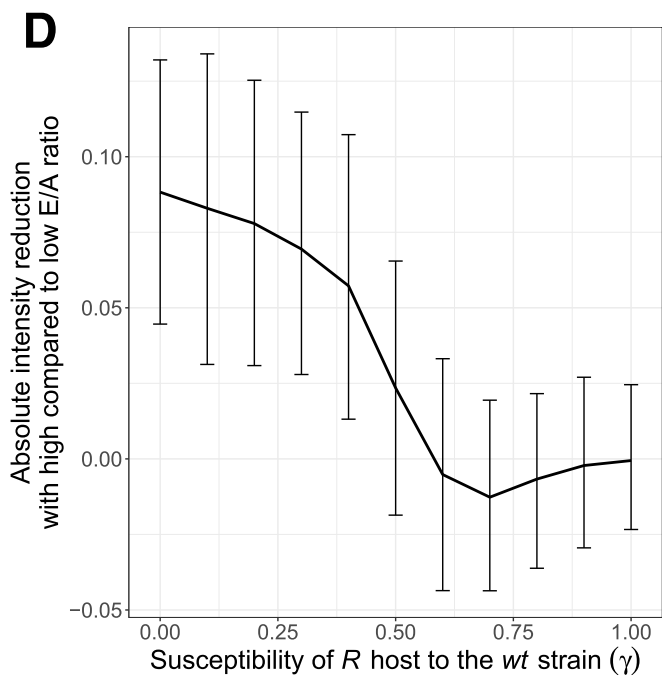

Absolute differences

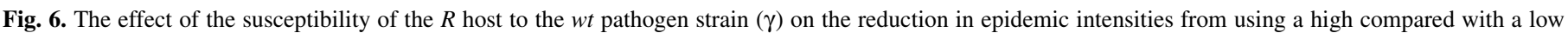

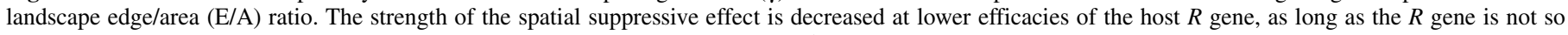

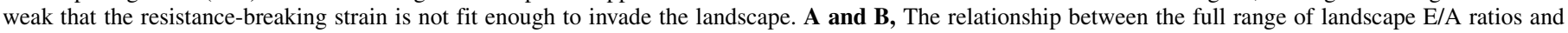

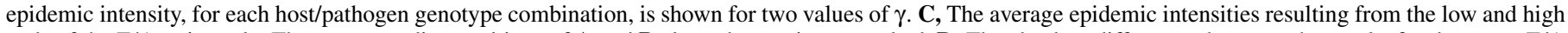

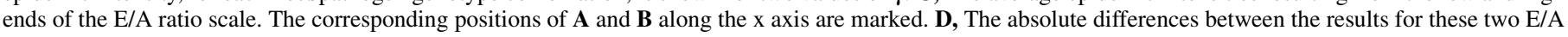

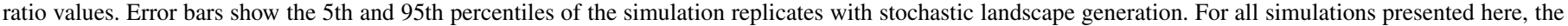
number of seasons $n_{y}=40$, the cost of the $r b$ trait $\delta=0.3$, and the kernel parameter $\eta=2$. 
seasons with larger values of $\delta$ because the greater trait cost means that the $r b$ strain spreads more slowly on the $R$ host, and takes longer to become competitive with the $w t$ strain on the $S$ host. There is no intermediate peak with $\delta=0.4$ (Fig. 8F) because the $r b$ trait is too costly for the $r b$ strain to spread as effectively on the $S$ host (Fig. 4C).

The variation in epidemic intensities, and in the strength of the spatial suppressive effect, due to the stochastic placement of fields in the landscape (size of the error bars), is generally highest at the points where the strength of the spatial suppressive effect is greatest (Figs. 5, 6, and 8). This implies that the effect of the specific stochastic arrangement of fields, and therefore the precise degree of spatial heterogeneity, is greatest when the general sensitivity to spatial dynamics is maximized.

\section{DISCUSSION}

Our model shows that planting susceptible and resistant host crop fields at smaller scales of spatial heterogeneity reduces epidemic intensities over a wide range of eco-evolutionary timescales. Such spatial strategies would therefore increase the durability of disease resistance, using a definition of durability analogous to the additional yield measured by the number of uninfected host growth days (van den Bosch and Gilligan 2003). This definition was also used in Fabre et al. (2012) and Fabre et al. (2015). There is of course variation in the definition of resistance durability, and alternative measures have been used in some other studies. These metrics include, the expected time until the invasion of a resistancebreaking strain, the time until this strain reaches a defined "takeover" threshold within the pathogen population (van den Bosch and Gilligan 2003), and the disease burden at a pathosystem's evolutionary equilibrium (Papaix et al. 2018). We however consider

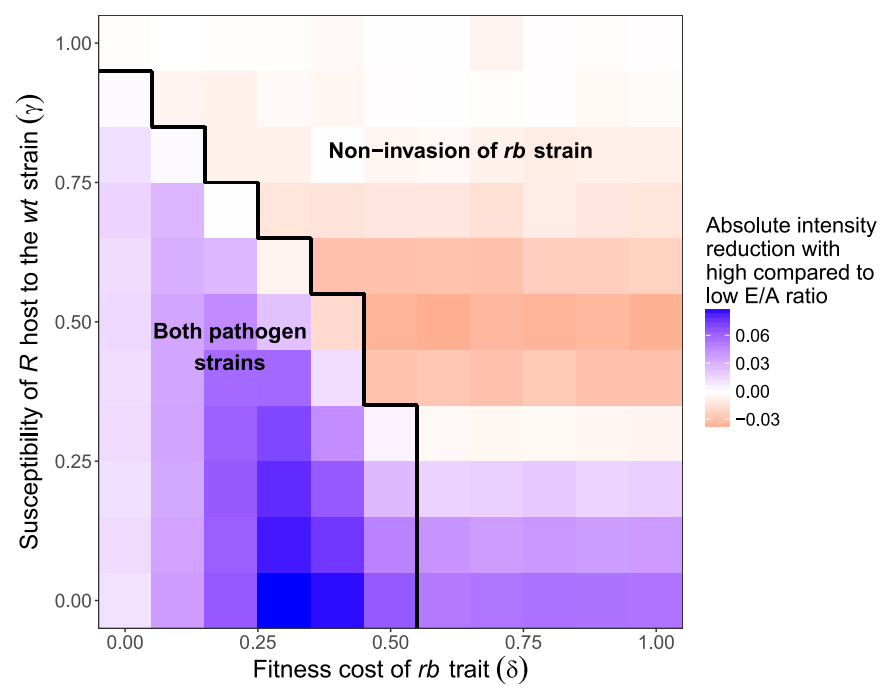

Fig. 7. The combined effects of the fitness cost of the resistance-breaking trait $(\delta)$, and the susceptibility of the $R$ host to the $w t$ pathogen strain $(\gamma)$, on the absolute reduction in epidemic intensities from using a high compared with a low landscape edge/area (E/A) ratio. The baseline epidemic intensities from using a low E/A ratio are shown in Supporting Figure S3. Note that using the proportional differences in epidemic intensity produces a qualitatively similar pattern. The strongest spatial suppressive effect occurs with a $100 \%$ effective resistance gene that imposes intermediate fitness costs on the resistancebreaking pathogen strain. The spatial effect operates in the reverse direction if the resistance-breaking strain is not fit enough to invade the landscape, so that only the wild-type strain is present, and the $R$ gene is of intermediate to lower efficacy. In the region where the $r b$ strain does not invade (defined arbitrarily as when the $r b$ epidemic intensity $<0.01$ ), there is no consistent response to changes in $\delta$ on the horizontal axis (due to the absence of the $r b$ strain). For all simulations presented here, the number of seasons $n_{y}=40$ and the kernel parameter $\eta=2$. that a metric based on the effective reduction in disease burden over a given time period, and therefore the "usefulness" of the deployed resistance, to be the most appropriate for our purposes.

The underlying mechanism behind the "spatial suppressive effect" on epidemic intensities is similar to the dilution effect that has been reported to reduce short term epidemic intensities in within field cultivar mixtures (Mundt 2002). Smaller scales of spatial heterogeneity reduce the local density of a given crop cultivar, meaning that some of a pathogen strain's potential force of infection is wasted as its inoculum disperses onto other nearby cultivars on which it has a lower reproductive fitness. This "wasted inoculum" effect generally suppresses the epidemics caused by each pathogen strain on the host cultivar where they are specialized and have the greatest fitness, while to a smaller extent boosting epidemics of that strain on their less preferred host. While this study has focused on qualitative plant resistance genes in a gene-for-gene system, these dynamics should be generally applicable to any system where multiple pathogen strains have different relative fitnesses when infecting multiple different host genotypes. Indeed, a similar basic spatial suppressive effect on resistance durability was observed by Papaix et al. (2018), in a model in which the resistance trait gradually eroded due to progressive small mutations in the pathogen population.

Our results also shed light on a number of the factors determining the strength of this spatial suppressive effect. This has significant implications for the potential effectiveness of any such spatial strategy if it were implemented by growers, as the benefit in terms of crop yield must outweigh any potential economic costs of farming in such a manner. Growing monoculture crops in very large fields, with consequentially large scales of spatial heterogeneity, is the norm in many systems of developed agriculture, largely for reasons of economic efficiency. It is therefore important that we characterize the fundamental eco-evolutionary processes that interact with host spatial structure, as these will ultimately play a crucial role in identifying the specific pathosystems where such spatial strategies are most likely to succeed.

The scale of pathogen dispersal within the agricultural landscape must correspond in some sense to the scale of heterogeneity implemented in that landscape in order to maximize the effectiveness of a spatial diversification strategy. Given that lower mean dispersal distances lead to the spatial suppressive effect being relevant over a wider range of scales (Fig. 3), pathogens with more restricted ranges of landscape scale dispersal are more likely to be effectively controlled in this manner. The underlying idea of requiring different spatial diversification strategies to control pathogens with different dispersal characteristics is supported by Sapoukhina et al. (2010). That work, however, focused on the qualitative differences between local short ranged dispersal through diffusion, and stratified dispersal that also included a separate long distance component, rather than looking at spatial heterogeneity as a continuous scale.

The spatial suppressive effect of cropping pattern on epidemic spread is maximized at an intermediate value for the fitness cost associated with the resistance-breaking trait (Fig. 5). This peak in effect strength is driven by two antagonistic effects that differ in their importance depending on whether fields in the landscape are mixed at small or large scales of spatial heterogeneity. The first of these is that increasing the fitness cost decreases the ability of the resistance-breaking strain to compete with the wild-type on the susceptible host. The second is that increasing the fitness cost also decreases the ability of the resistance-breaking strain to survive on the resistant host. The first effect is more important at smaller spatial scales of heterogeneity, while the second effect is more important at larger scales. The intermediate peak occurs when the increasing fitness cost causes the second effect to increase in relative impact such that it outweighs the impact of the first effect. The exact fitness cost value for this peak in the spatial suppressive effect is lower with a less effective resistance gene (Fig. 7). 
A less effective resistance gene lowers the strength of the spatial suppressive effect, as long as the resistance-breaking strain has a high enough fitness to be able to invade the agricultural landscape (Figs. 6 and 7). Whether or not the resistance-breaking strain can invade a landscape depends on both its associated fitness costs and on the efficacy of the resistance gene. If invasion does not occur, and only the wild-type strain is present, intermediate to lower efficacy $R$ genes can drive a reverse spatial suppressive effect, where epidemic intensities are higher with smaller scales of spatial heterogeneity. This occurs because wild-type epidemics are only able to sustain themselves in partially resistant fields when there are nearby susceptible fields to act as inoculum sources. The effect is similar to that observed by Papaix et al. (2014), who showed in a single strain system that the directional effect of spatial aggregation depended on the $R_{0}$ value for the disease epidemic on the resistant variety.

The combined genetic context in this system is created by the combination of the fitness cost of the resistance-breaking trait and the efficacy of the resistance gene. From this we can conclude that a spatial diversification strategy is most likely to be cost effective when using a $100 \%$ effective major resistance gene that imposes intermediate fitness costs on a resistance-breaking pathogen strain. Despite this, a spatial strategy is still likely to be at least partially effective in any genetic context, as long as the resistance-breaking pathogen strain is fit enough to invade and persist within the landscape (Fig. 7). The fact that spatial diversification can actually worsen epidemics when only a wild-type strain is present, and a partially effective resistance gene is used, highlights the necessity of understanding the state of the pathogen community and the genetic nature of the system before implementing such control strategies. A spatial strategy will be less effective when there are no fitness costs for the resistance-breaking strain, however an effect is still observed due to the time required for this strain to fully take over the pathogen population. This naturally becomes particularly apparent when looking over a lower number of seasons.

It may be possible to obtain experimental estimates for the parameters in our model from published studies, however there is often an inherent difficulty in applying data from a laboratory setting to a complex real world epidemic (Cunniffe and Gilligan 2020). A more reliable approach would be to estimate the infection rate from disease progress data taken from a field study. The fitness costs of the resistance-breaking trait could be estimated using methods such as that described by Montarry et al. (2010). These authors showed that the while the fitness costs associated with virulence in Phytophthora infestans were generally low, they were
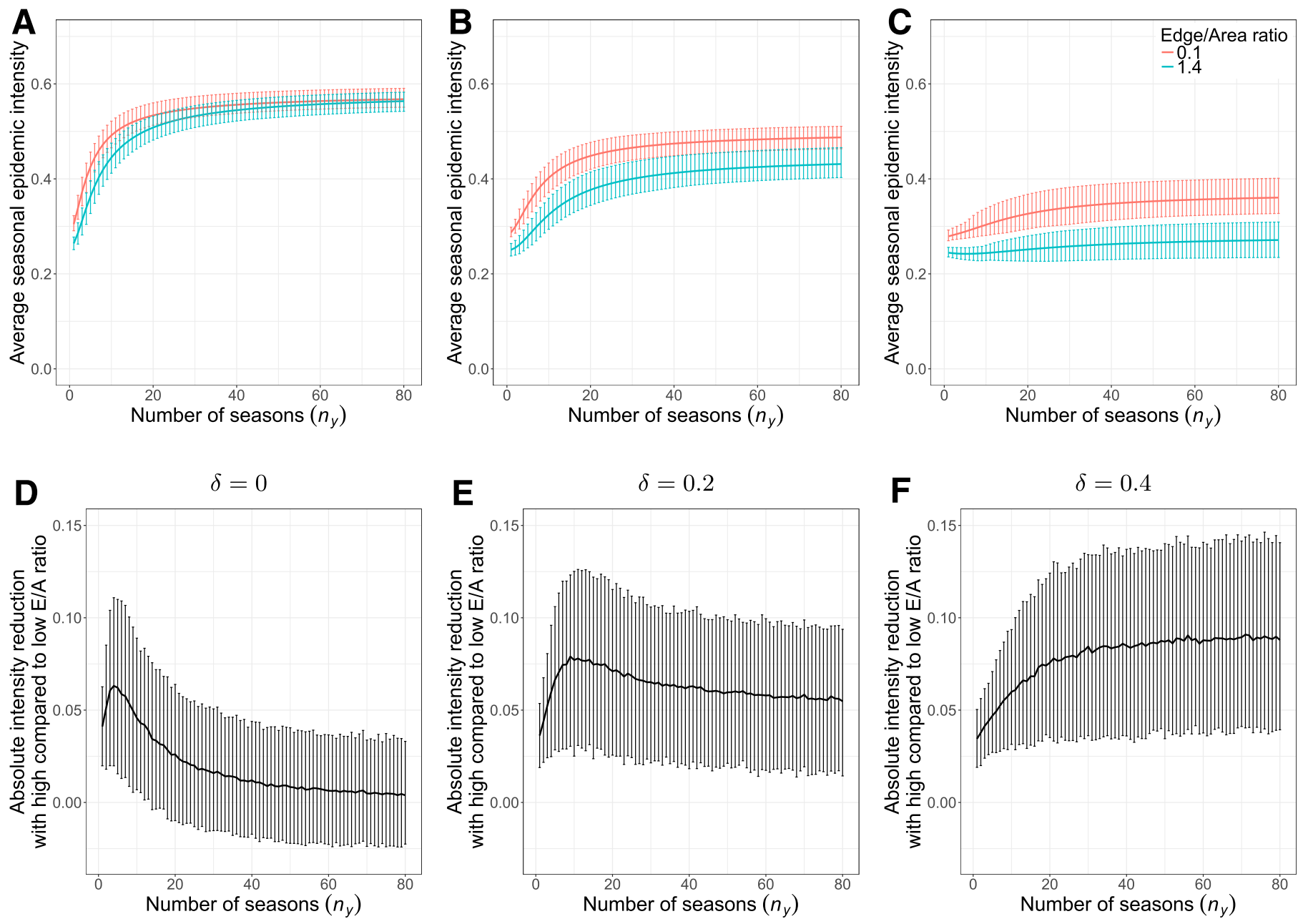

$$
\delta=0
$$$$
\delta=0.2
$$$$
\delta=0.4
$$

Fig. 8. The effect of the number of seasons $\left(n_{y}\right)$ on the reduction in epidemic intensities from using a high compared with a low landscape edge/area (E/A) ratio, at different fitness costs of the resistance-breaking trait $(\delta)$. The strength of the spatial suppressive effect depends on the length of the time frame of interest. There is the potential for a peak effect strength over an intermediate number of seasons, which occurs over a greater number of seasons with a higher fitness cost of the resistance-breaking trait. A, B, and C, The average epidemic intensities resulting from the low and high ends of the E/A ratio scale. D, E, and F, The absolute differences between the results for these two E/A ratio values. Error bars show the 5th and 95th percentiles of the simulation replicates with stochastic landscape generation. For all simulations presented here, the $R$ host is completely resistant to the $w t$ strain $\gamma=0$ and the kernel parameter $\eta=2$. 
cumulative as the number of virulence factors was increased. This implies that the higher fitness costs studied in our model would be relevant to scenarios where the pathogen population requires multiple virulence factors to overcome a combination of resistance genes in the host plant. Resistance efficacies can be measured by methods such as comparing the area under the disease progress curve for different epidemics, although again to infer the value of a parameter in our model, some model fitting would be required (Griffey et al. 1993; Jeger and Viljanen-Rollinson 2001). For the purpose of suggesting potential pathosystems where a spatial diversification strategy is more likely to be effective, there is currently a shortage of available useful data. While resistancebreaking/virulence fitness costs, or partial resistance gene efficacies, have been commonly inferred, they have only very rarely been calculated using the methods described above that would allow their inclusion in an epidemiological model.

There is also a lack of available field or experimental data that could be used to generally parameterize the models presented in this study, and validate the dynamics that we have described. We would require disease progress data, separated by host cultivar/pathogen strain combination, for epidemics in host landscapes with a range of cropping ratios and scales of spatial heterogeneity. For larger pathogen dispersal scales, these data could be obtained by conducting field trials; however, this may be impractical due to the number and scale of trials that would be required. For this reason, it might be more practical to conduct greenhouse experiments on a plant disease system with a pathogen that disperses at smaller spatial scales. The usefulness of such data for validation purposes would be increased if a number of different resistance traits, with different associated pathogen fitness costs, could be tested.

A critical factor that is generally neglected within the study of resistance durability is the length of the time frame of interest. If we consider any improvement in durability to be the yield gain achieved, this will naturally depend on the time period over which we measure such gains, which in practice should itself depend on factors such as the frequency with which new resistant cultivars are developed. This timescale will obviously vary for different crop disease systems, and will play a significant role in determining whether a spatial strategy has a large enough effect to be economical for practical use. The strength of the spatial suppressive effect depends on the number of seasons over which it is measured, with the potential for a peak in spatial suppressive effect strength over an intermediate number of seasons (Fig. 8), i.e., in between a short term epidemiological timescale and the long term evolutionary equilibrium. In a similar manner to the effect of the fitness cost of the resistance-breaking trait, this occurs because the different infection classes in the system respond at different and varying rates to changes in season number. The frequency of these host-pathogen genotype combinations, and therefore the effect they have on overall epidemic intensity, depends on the scale of spatial heterogeneity in the distribution of host genotypes within the landscape. The resultant peak spatial suppressive effect occurs over a higher number of seasons with a higher cost of the resistancebreaking trait, due to the slower spread rate of this resistancebreaking strain. Generally this means that a spatial strategy is most likely to be effective over short timescales for resistance-breaking strains that carry little or no fitness costs, and over longer timescales for more costly traits.

Although the main focus of this study has been the effect of spatial heterogeneity at the landscape between-field scale, there is no reason why the model could not be applied to smaller spatial scales. Application of the model to specific pathosystems at any spatial scale would require careful considerations of both the relative scales of pathogen dispersal and the proportion of inoculum that disperses within and between local "host groups". These host groups, which we generally refer to as "fields" in the current study, could instead represent smaller subunits of fields or even individual plants at smaller spatial scales. It may also be important to consider differences in density dependence effects at different spatial scales (van den Bosch and Gilligan 2008).

In our study, the initial frequency of the resistance-breaking strain in the pathogen population could represent the background equilibrium frequency resulting from a mutation-selection balance in the absence of positive selection from the deployment of the resistant host (Fabre et al. 2012). However, unlike Fabre et al. (2012) we assumed this initial frequency took a fixed value, and did not depend on the fitness cost of the resistance-breaking pathogen. It would instead be possible to include the fitness cost in setting the initial frequency, with pathogen strains with higher fitness costs starting at a lower initial frequency. Our assumption was motivated by simplicity, since-unlike Fabre et al. (2012)-we modeled the resistance-breaking and wild-type pathogen strains separately, greatly reducing the importance of the mutation-selection balance in setting their relative densities throughout the entire epidemic. We note it is possible to infer from the results presented here the likely effect of making the initial frequency of a resistance-breaking pathogen strain depend on its fitness cost. Were such an assumption to be included, any pathogen with a higher fitness cost would simply start at a lower initial frequency, and so spread more slowly. This would merely exaggerate the effect of having a higher fitness cost. We also note in passing that there is some evidence, for single nucleotide substitutions in Tobacco etch virus, that higher fitness cost mutants can be much more common than those with a lower fitness cost in experimental systems (Carrasco et al. 2007). This suggests that naive application of the mutation-selection balance, assuming a fixed mutation rate, may not be representative of real biological systems.

The frequency $\theta=0.01$ used in our study is relatively high compared with some estimates for rare pathogen mutants under a mutation-selection balance. These frequencies can be several orders of magnitude lower depending on the number of required number of mutational events (Ribeiro et al. 1998). However, the fitness cost of a resistance-breaking mutation might be very low (or even zero) on the reservoir host, meaning that relatively large initial frequencies originating from this alternate host may be plausible. We also note that for management of resistance to be sufficiently important to growers for them to even consider the types of landscape scale reorganization described here, any resistance-breaking strain must have reached a sufficiently high frequency to be detectable. Only once detection occurs will the maximization of resistance durability, as measured by long term epidemic intensity, become a priority. Using a lower initial frequency of the resistance-breaking strain would be very unlikely to affect the qualitative behavior of our model, and would instead mean that the equilibrium is simply reached after a greater number of seasons. Our model could be further refined however by using a separate pathogen death rate term, combined with higher infection rates, such that the resistancebreaking strain still spreads rapidly with the use of a lower initial frequency.

The dynamics in our model have been explored with a single set of transmission related parameters, which characterize a baseline epidemic scenario where only a susceptible host variety is planted in the landscape, and only the wild-type pathogen strain is present. This was done in order to reduce the number of freely varying parameters, so that the already complex set of interactions in the model could be studied in greater depth. The fact that none of the above mentioned transmission related parameters have a directly differential effect on either pathogen strain, or on either host variety, makes them less of a priority to explore here. The effects of varying these parameters to produce different baseline epidemics, with which the effects of resistance deployment can be compared, have already been explored in the nonspatially explicit model of Fabre et al. (2012). In our model however, we expect that increasing the proportion of between field transmission would increase the strength of the spatial suppressive effect, while increasing the 
contribution of the reservoir host to disease transmission would results in slower spread of the resistance-breaking pathogen over time. This latter prediction is due to the fact that in our model the genetic composition of the pathogen population in the reservoir host is generally more stable than it is in the primary host fields. Modifying the stability of the reservoir host pathogen population in the current model would simply change the rate at which the resistance-breaking pathogen strain spreads.

In order to gain a more intuitive understanding of how the dynamics observed within our model operate, it would be a valuable approach to investigate the potential for representing them in simpler mathematical terms. The classical approach of models for within field cultivar mixtures could be applied to this by comparing pure versus mixed cultivar stands in an otherwise nonspatial model (Jeger et al. 1981). Unfortunately, both the model presented in this study and that of Fabre et al. (2012) are too complex for the mathematical analysis of this approach to be usefully carried out. Furthermore, the focus of such an approach on the extreme ends of the spatial heterogeneity scale would make comparisons with the more realistic intermediate scales of heterogeneity used in our study difficult. More recent (currently unpublished) work we have undertaken on this topic has however resulted in the development of a spatially implicit model that captures many of the fundamental dynamics described in this paper. The simpler nature of this new mathematical model, with a reduced number of parameters, allows the representation of any scale of spatial heterogeneity combined with an independently variable cropping ratio. This simpler model also allows a useful degree of mathematical analysis.

In the current study we have restricted the cropping ratio of the susceptible and resistant cultivars to 50:50, in order to avoid having to consider potential interactions between the effects of the scale of spatial heterogeneity and the amount of resistant crop deployed. The potential ways in which the patterns we have described might be influenced by different cropping ratios is a valid area for further study, as is the way that optimal cropping ratios might in turn be influenced by spatial dynamics. The nonspatial model of Fabre et al. (2012) demonstrated that the optimal cropping ratio (i.e., the proportion of resistant fields) varied from intermediate to high values, and depended among other factors on the relative contributions of within field, between field and reservoir-driven infection. Demographic stochasticity, which has been shown to bias optimal cropping ratios toward higher values, is another potential route for further investigation (Lo Iacono et al. 2013). The associated chance of pathogen strain extinction at low frequencies or under periodic perturbation could potentially interact with the effects of patch size and spatial heterogeneity on disease dynamics.

There have been a number of studies on the maintenance of qualitative disease resistance in plants that focus on coevolutionary dynamics and the factors that promote balanced polymorphisms within pathogen populations (Leonard 1977; Tellier and Brown 2007). Given that these models feature host genotype frequencies that oscillate in response to changes in the pathogen population, they are highly relevant to natural systems, but say little for the agricultural systems that we are concerned with, where variety frequencies are controlled directly by the grower.

In conclusion, this study has demonstrated the key effect that spatial structure can have on disease resistance durability. The diversification of resistance genes at small scales of spatial heterogeneity is a potentially valuable strategy for improving long term crop yields, depending on whether the strength of the spatial suppressive effect leads to such a strategy being economical. Factors such as the pathogen dispersal scale, the genetic properties of the host-pathogen interaction, and the time frame of interest play a crucial role, and highlight the need for a thorough understanding of any disease system to which this strategy is applied.

\section{ACKNOWLEDGMENTS}

We thank Elliott Bussell, Richard Stutt, Andrew Craig, and Eleftherios Avramidis for helpful discussions and technical advice related to this project.

\section{LITERATURE CITED}

Bousset, L., Sprague, S. J., Thrall, P. H., and Barrett, L. G. 2018. Spatiotemporal connectivity and host resistance influence evolutionary and epidemiological dynamics of the canola pathogen Leptosphaeria maculans. Evol. Appl. 11:1354-1370.

Brown, J. K. M. 1995. Recombination and selection in populations of plant pathogens. Plant Pathol. 44:279-293.

Bruns, E., Carson, M. L., and May, G. 2014. The jack of all trades is master of none: A pathogen's ability to infect a greater number of host genotypes comes at a cost of delayed reproduction. Evolution 68:2453-2466.

Burdon, J. J., and Thrall, P. H. 2008. Pathogen evolution across the agro-ecological interface: Implications for disease management. Evol. Appl. 1:57-65.

Burdon, J. J., Zhan, J., Barrett, L. G., Papaï, J., and Thrall, P. H. 2016. Addressing the challenges of pathogen evolution on the world's arable crops. Phytopathology 106:1117-1127.

Carrasco, P., de la Iglesia, F., and Elena, S. F. 2007. Distribution of fitness and virulence effects caused by single-nucleotide substitutions in tobacco etch virus. J. Virol. 81:12979-12984.

Cui, H., Tsuda, K., and Parker, J. E. 2015. Effector-triggered immunity: From pathogen perception to robust defense. Annu. Rev. Plant Biol. 66:487-511.

Cunniffe, N. J., and Gilligan, C. A. 2020. Use of mathematical models to predict epidemics and to optimise disease detection and management. Chapter 12 in: Emerging Plant Diseases and Global Food Security. J. Ristaino, ed. American Phytopathological Society, St. Paul, MN.

Djidjou-Demasse, R., Moury, B., and Fabre, F. 2017. Mosaics often outperform pyramids: Insights from a model comparing strategies for the deployment of plant resistance genes against viruses in agricultural landscapes. New Phytol. 216:239-253.

Fabre, F., Rousseau, E., Mailleret, L., and Moury, B. 2012. Durable strategies to deploy plant resistance in agricultural landscapes. New Phytol. 193: 1064-1075.

Fabre, F., Rousseau, E., Mailleret, L., and Moury, B. 2015. Epidemiological and evolutionary management of plant resistance: Optimizing the deployment of cultivar mixtures in time and space in agricultural landscapes. Evol. Appl. 8:919-932.

Flor, H. H. 1971. Current status of the gene-for-gene concept. Annu. Rev. Phytopathol. 9:275-296.

Fraile, A., Pagan, I., Anastasio, G., Saez, E., and Garcia-Arenal, F. 2011. Rapid genetic diversification and high fitness penalties associated with pathogenicity evolution in a plant virus. Mol. Biol. Evol. 28:1425-1437.

Garcia-Arenal, F., and McDonald, B. A. 2003. An analysis of the durability of resistance to plant viruses. Phytopathology 93:941-952.

Griffey, C., Das, M., and Stromberg, E. 1993. Effectiveness of adult-plant resistance in reducing grain yield loss to powdery mildew in winter wheat. Plant Dis. 77:618-622.

Harrison, B. D. 2002. Virus variation in relation to resistance-breaking in plants. Euphytica 124:181-192.

Jeger, M., Griffiths, E., and Jones, D. G. 1981. Disease progress of nonspecialised fungal pathogens in intraspecific mixed stands of cereal cultivars. I. Models. Ann. Appl. Biol. 98:187-198.

Jeger, M., and Viljanen-Rollinson, S. 2001. The use of the area under the disease-progress curve (AUDPC) to assess quantitative disease resistance in crop cultivars. Theor. Appl. Genet. 102:32-40.

Johnson, R. 1979. Concept of durable resistance. Phytopathology 69:198-199.

Knott, D. 1961. The inheritance of rust resistance. vi. The transfer of stem rust resistance from Agropyron elongatum to common wheat. Can. J. Plant Sci. 41:109-123.

Kolmer, J. 1996. Genetics of resistance to wheat leaf rust. Annu. Rev. Phytopathol. 34:435-455.

Lannou, C., and Mundt, C. C. 1996. Evolution of a pathogen population in host mixtures: Simple race-complex race competition. Plant Pathol. 45:440-453.

Leonard, K. 1977. Selection pressures and plant pathogens. Ann. N.Y. Acad. Sci. 287:207-222.

Lo Iacono, G., van den Bosch, F., and Gilligan, C. A. 2013. Durable resistance to crop pathogens: An epidemiological framework to predict risk under uncertainty. PLOS Comput. Biol. 9:e1002870.

Madden, L. V., Hughes, G., and van den Bosch, F. 2007. The Study of Plant Disease Epidemics. American Phytopathological Society, St. Paul, MN

Mailleret, L., and Lemesle, V. 2009. A note on semi-discrete modelling in the life sciences. Philosophical Transactions of the Royal Society A-Mathematical Physical and Engineering Sciences 367:4779-4799. 
McDonald, B. A., and Linde, C. 2002. Pathogen population genetics, evolutionary potential, and durable resistance. Annu. Rev. Phytopathol. 40: 349-379.

Montarry, J., Hamelin, F. M., Glais, I., Corbière, R., and Andrivon, D. 2010. Fitness costs associated with unnecessary virulence factors and life history traits: Evolutionary insights from the potato late blight pathogen Phytophthora infestans. BMC Evol. Biol. 10:283.

Mundt, C. C. 2002. Use of multiline cultivars and cultivar mixtures for disease management. Annu. Rev. Phytopathol. 40:381-410.

Papaï, J., David, O., Lannou, C., and Monod, H. 2013. Dynamics of adaptation in spatially heterogeneous metapopulations. PLoS One 8:e54697.

Papaï, J., Rimbaud, L., Burdon, J. J., Zhan, J., and Thrall, P. H. 2018. Differential impact of landscape-scale strategies for crop cultivar deployment on disease dynamics, resistance durability and long-term evolutionary control. Evol. Appl. 11:705-717.

Papaï, J., Touzeau, S., Monod, H., and Lannou, C. 2014. Can epidemic control be achieved by altering landscape connectivity in agricultural systems? Ecol. Modell. 284:35-47.

Parnell, S., Gottwald, T., Cunniffe, N., Alonso Chavez, V., and van den Bosch, F. 2015. Early detection surveillance for an emerging plant pathogen: a rule of thumb to predict prevalence at first discovery. Proc. R. Soc. B 282: 20151478.

Parnell, S., Gottwald, T., Gilligan, C., Cunniffe, N., and van den Bosch, F. 2010. The effect of landscape pattern on the optimal eradication zone of an invading epidemic. Phytopathology 100:638-644.

Parnell, S., Gottwald, T., van den Bosch, F., and Gilligan, C. 2009. Optimal strategies for the eradication of Asiatic citrus canker in heterogeneous host landscapes. Phytopathology 99:1370-1376.

Plantegenest, M., Le May, C., and Fabre, F. 2007. Landscape epidemiology of plant diseases. J. R. Soc. Interface 4:963-972.

Qureshi, N., Kandiah, P., Gessese, M. K., Nsabiyera, V., Wells, V., Babu, P., Wong, D., Hayden, M., Bariana, H., and Bansal, U. 2018. Development of co-dominant kasp markers co-segregating with ug99 effective stem rust resistance gene sr26 in wheat. Mol. Breed. 38:97.
Ribeiro, R. M., Bonhoeffer, S., and Nowak, M. A. 1998. The frequency of resistant mutant virus before antiviral therapy. AIDS 12:461-465.

Rimbaud, L., Papaix, J., Barrett, L. G., Burdon, J. J., and Thrall, P. H. 2018. Mosaics, mixtures, rotations or pyramiding: What is the optimal strategy to deploy major gene resistance? Evol. Appl. 11:1791-1810.

Sapoukhina, N., Durel, C.-E., and Le Cam, B. 2009. Spatial deployment of gene-for-gene resistance governs evolution and spread of pathogen populations. Theor. Ecol. 2:229-238.

Sapoukhina, N., Tyutyunov, Y., Sache, I., and Arditi, R. 2010. Spatially mixed crops to control the stratified dispersal of airborne fungal diseases. Ecol. Modell. 221:2793-2800.

Skelsey, P., Rossing, W. A. H., Kessel, G. J. T., and van der Werf, W. 2010. Invasion of Phytophthora infestans at the landscape level: How do spatial scale and weather modulate the consequences of spatial heterogeneity in host resistance? Phytopathology 100:1146-1161.

Skelsey, P., With, K. A., and Garrett, K. A. 2013. Why dispersal should be maximized at intermediate scales of heterogeneity. Theor. Ecol. 6: 203-211.

Stam, R., and McDonald, B. A. 2018. When resistance gene pyramids are not durable_-The role of pathogen diversity. Mol. Plant Pathol. 19:521-524.

Tellier, A., and Brown, J. K. M. 2007. Stability of genetic polymorphism in host-parasite interactions. Proc. R. Soc. B 274:809-817.

Thrall, P. H., and Burdon, J. J. 2003. Evolution of virulence in a plant host-pathogen metapopulation. Science 299:1735-1737.

van den Bosch, F., and Gilligan, C. A. 2003. Measures of durability of resistance. Phytopathology 93:616-625.

van den Bosch, F., and Gilligan, C. A. 2008. Models of fungicide resistance dynamics. Annu. Rev. Phytopathol. 46:123-147.

Wolfe, M., and McDermott, J. 1994. Population genetics of plant pathogen interactions: The example of the Erysiphe graminis-Hordeum vulgare pathosystem. Annu. Rev. Phytopathol. 32:89-113.

Zhan, J., and McDonald, B. A. 2013. Field-based experimental evolution of three cereal pathogens using a mark-release-recapture strategy. Plant Pathol. 62:106-114. 